\title{
The Space Weather Monitor Project: bringing hands-on science to students of the developing world for the IHY2007
}

\author{
Deborah Scherrer $^{1}$, M. Cristina Rabello-Soares ${ }^{1}$ \\ and Cherilynn Morrow ${ }^{2}$ \\ ${ }^{1}$ Stanford University, Solar Physics, HEPL-4085, Stanford, CA 94305-4085, USA, \\ dscherrer@solar.stanford.edu \\ ${ }^{2}$ Space Science Institute, 4750 Walnut Street, Suite 205, Boulder, Colorado 80301, USA, \\ morrow@SpaceScience.org
}

\begin{abstract}
Stanford's Solar Center, Electrical Engineering Department, and local educators have developed inexpensive Space Weather Monitors that students around the world can use to track solar- and lightning-induced changes to the Earth's ionosphere. Through the United Nations Basic Space Science Initiative (UNBSSI) and the International Heliophysical Year (IHY) Education and Public Outreach programme, our Monitors are being deployed to 191 countries. In partnership with Chabot Space and Science Center, we are designing and developing classroom and educator support materials to accompany the distribution. Materials will be culturally sensitive and will be translated into the six official languages of the United Nations (Arabic, Chinese, English, French, Russian, and Spanish). Monitors will be provided free of charge to developing nations and can be set up anywhere there is access to power.
\end{abstract}

Keywords. education, Sun, solar-terrestrial relations, Earth, miscellaneous

\section{Introduction}

Earth's ionosphere reacts strongly to the intense X-ray and ultraviolet radiation released by the Sun during solar events and by lightning during thunderstorms. Imagine students being able to track these sudden ionospheric disturbances by using a receiver to monitor the signal strength from distant VLF transmitters and noting unusual changes as the waves bounce off the ionosphere. Stanford's Solar Center, Electrical Engineering Department, and local educators have developed inexpensive Space Weather Monitors that students around the world can use to track changes to the Earth's ionosphere. Two versions of the monitors exist - a low-cost version nicknamed SID designed to detect solar flares; and a more sensitive version (AWESOME) that provides both solar and nighttime research-quality data.

Through the United Nations Basic Space Science Initiative (UNBSSI) and the IHY International Education and Public Outreach programme, we expect to deploy, without charge, our monitors to high school-equivalents and universities in developing nations of the world for IHY 2007. Monitors are inexpensive (US\$200 for the student version; US $\$ 2400$ for the research quality). The monitors come preassembled, but students buy in by designing and building their own antenna, costing little and taking a few hours to assemble. Participants also provide a simple PC to record the data and, if possible, an internet connection to share their data with the rest of the worldwide team. The monitors can be set up anywhere there is access to power.

The IHY is ideally suited to support a global VLF receiver venture. This project directly relates to the goals of IHY by advancing our understanding of the fundamental 
heliophysical processes that govern the Sun, Earth and heliosphere and demonstrating the beauty, relevance and significance of space and Earth science to the world. As a result of IHY, AWESOME (research-quality) receivers have recently been set up successfully in Ireland, Tunisia, India, Algeria, and Morocco. Seventy of the inexpensive SIDs have already been distributed to the USA and a dozen more countries.

Our project enables researchers, educators, and students to produce scientific data from their own instrument and access data from other worldwide team members. It provides tools for doing research, both independently and collaboratively. A 2002 decadal survey by the USA National Research Council noted that "understanding and monitoring the fundamental processes responsible for solar-terrestrial coupling are vital to being able to fully explain the influence of the Sun on the near-Earth environment". Abundant evidence shows that high school and introductory college science teaching is improved when the students engage in doing real science on real data.

\section{Students collect and analyze scientific data}

Our monitors capture signal strength from ELF/VLF frequencies between about $30 \mathrm{~Hz}-$ $50 \mathrm{kHz}$, transmitted by nations to communicate with their submarines. SID data are easy to read and understand, thus readily accessible to introductory college and high school groups. In addition to solar flare phenomena, the graphs host a wealth of details about the Earth's ionosphere and how it changes during the day/night cycle, from season to season, and how it responds to lightning storms and other ionospheric events.

Data recorded by our instruments resemble seismograph data (Figure 1). Students receive their SID data as a signal strength value and a timestamp. The data collected are easily read and graphed either by Excel or a gnu-based plot programme provided. Solar events show up as spikes in the signal strength. The more sensitive AWESOME monitors allow students to track nighttime lightning phenomena as well.

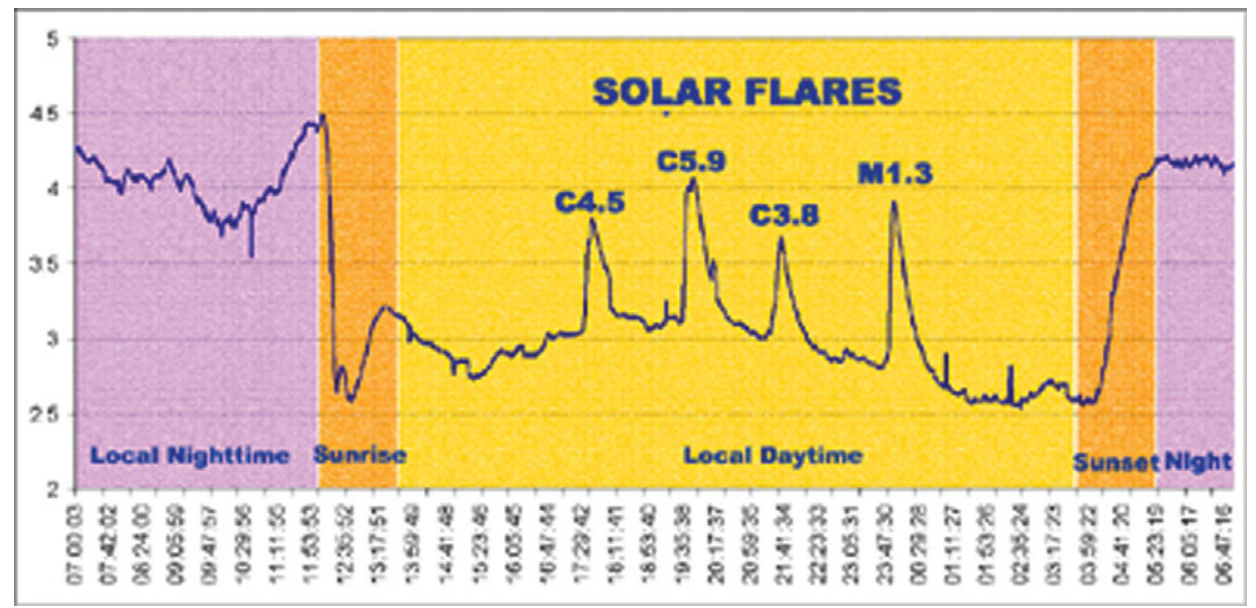

Figure 1. Sample SID data.

Stanford hosts a centralized data repository for both SID and AWESOME data. (http://sid.stanford.edu/database-browser/) Data are useful for solar researchers, for ionospheric researchers, and for educators and students, even those not hosting monitors. Observations are highly appropriate for creative use in student research. We suggest to students (http://solar-center.stanford.edu/SID/educators/) a collection of 
potential research projects relating to the Sun and space weather that will encourage their participation in hands-on research and collaboration, giving them experience in a field of vital worldwide interest. Research topics relate to:

- Sunrise and sunset ionization phenomena;

- Tracking solar storms back to the Sun;

- Understanding ionization effects;

- Nighttime research phenomena;

- Using ionization changes as earthquake predictors.

Student researchers with access to the internet can compare their data with that from NOAA's GOES satellites to help identify solar flares. Students using a monitor prototype have already identified flares that GOES "missed" due to human error. Students can track events back to specific active regions on the Sun and can view corresponding images from solar observatories such as SOHO/MDI, SOHO/EIT, TRACE, and eventually SDO. Students can also compare their data with solar-induced effects on radio communications, high-flying aircraft, satellites, even pigeon racing. As an advanced research project, they might attempt to predict possible flare sites using MDI's farside imagery. (See http://soi.stanford.edu/data/full_farside). Students using prototype monitors have also attempted to correlate their solar events with environmental changes on Earth. In most of their research, students benefit by talking with other students and comparing data from other sites. The programme provides student access to Scientist Mentors to assist in undertaking and understanding their research.

\section{Educational components}

In partnership with Chabot Space and Science Center of Oakland, California, USA we are designing, developing, testing, and assessing classroom and educator support materials to accompany the distribution. Curriculum materials, designed and tested by Master Teachers, will be inquiry-based and professionally assessed. Online and DVD/video teacher training will be available to support teachers around the world. Extensive background and accompanying materials will be provided, both on the web and in hard-copy form. A centralized database and communications hub have already been set up by Stanford for communication amongst teachers and students worldwide.

This project is closely aligned with the USA National Academy of Science/National Research Council's National Science Education Standards (National Research Council, 1996). The Standards emphasize science as inquiry. Unifying concepts and processes parallel those in the Standards, and include focus on the fundamental aspects of solar activity and their effects on the Earth, on obtaining data, taking measurements, on observing and understanding change and constancy, and learning to interpret and explain results from data. The pairing of the instrument with scientific inquiry improves understanding of technological design and the functions technology can play in science. As the Standards encourage, the project facilitates teachers and students working directly with scientists and real data, providing them unique opportunities to experience science as a human endeavor and giving them direct insight into the nature of scientific knowledge. The activities developed for the Teachers Guides reflect "best practices" in education as experienced and interpreted by Master Teachers and include specific information about alignment with the National Science Standards. Although we understand that the US National Science Standards do not apply to other nations, we feel the concepts and guiding principles are strong and valid models for designing science education throughout the world. 
To the extent possible, materials will be reviewed for cultural appropriateness and a subset will be translated into the six official languages of the United Nations (Arabic, Chinese, English, French, Russian, and Spanish).

Blindness is a serious problem in many developing nations, and often blind students are "left behind" in the education process. We are looking for funding for a special version of the education materials to be adapted for the blind, translated into Braille, and made available to any country upon request. SID data will be sonified to support equal access by blind students. Curricula support materials such as NASA's/Noreen Grise's Touch the Sun book (Grice, 2005) will also be included in this package.

The programme includes teacher and student access to Scientists Mentors for discussions about problems, data, and research. Scientist Mentors provide direct connections between students, teachers, and the international research community. Loosely modeled on JPL's Solar System Ambassador programme (http: //www2 .jpl .nasa.gov/ambassador), we are already experimenting with soliciting and using mentors - motivated individuals with scientific skill and experience in solar physics, geophysics, ionospheric physics, or radio. Mentor training, also modeled after the JPL programme, will be provided through teleconferences, the web, and supplied materials. Once our full Scientist Mentor programme is in place, we will publish and make available to sites a database (via web, email, or direct mailing) of Mentors' expertise, skills, interests, languages, and contact information. SID sites will be encouraged to build a relationship with a Scientist Mentor who has the skills necessary to solve their problems or answer their questions. Mentors will also serve as role models for Science-Technology-Engineering-Math (STEM) careers. Part of mentor training will provide information on encouraging students to consider STEM careers, no matter where in the world they might live.

\section{Conclusions}

The International Heliophysical Year aims to explore the response of the terrestrial atmosphere and magnetosphere to external drivers. The future of exploration lies in global understanding of the ways that the Sun affects Earth's environment. We propose a combined educational and research programme aimed at developing nations and featuring a network of sensors distributed to a worldwide team who will perform quantitative comparisons of ionospheric disturbances. The network consists of state-of-the-art ELF/VLF receivers sensitive to a broad range of ionospheric phenomena and student-appropriate low cost space-weather sensors sensitive to solar-flare-induced sudden ionospheric disturbances. Educational materials accompany the distribution and access to Scientist Mentors assures adequate student support. For more information about the Space Weather Monitor programme, see http://solar-center.stanford.edu/SID/.

\section{Acknowledgements}

This programme was originally developed and funded by NSF's Center for Integrated Space Weather Modeling. Additional funding was provided by NASA grant NAG53077, supporting the MDI instrument onboard the SOlar and Heliospheric Observatory (SOHO). SOHO is a project of international cooperation between ESA and NASA.

\section{References}

Grice, N. 2005, Touch the Sun - A NASA Braille Book. Joseph Henry Press (an imprint of the National Academies Press), Washington, DC

National Research Council 1996, National Science Education Standards. National Academies Press, Washington, DC 